\title{
"Nunc eo ingenio sum." Philologie et écriture de soi dans les Variae lectiones de Marc-Antoine Muret
}

\author{
LUCIE CLAIRE
}

The collection Variae lectiones by Marc-Antoine Muret (1526-1585), which is part of the humanist tradition of miscellanea, is characterized by the prominent presence of the first person. This article proposes to study the relationship between philology and writing about oneself by examining the different kinds of "I" used by Muret. Staking at the outset a claim to auctoritas, the "I" of Variae lectiones refers above all to a scholarly and erudite persona, which the French humanist develops with the ambition of consolidating his personal reputation at a crucial period of his career in Italy. Finally, while writing as a philologist, Muret sometimes reveals himself through his readings of the Ancients, and hints at a more intimate "I" which emerges in some accounts of episodes experienced.

Dans la préface qui précède les huit premiers livres de ses miscellanées philologiques, les Variae lectiones, Marc-Antoine Muret décrit le lien profond qui l'unit aux textes de l'Antiquité. ${ }^{2}$ Au moment d'exposer à son protecteur, le cardinal Hippolyte d'Este, les raisons qui ont motivé la

1 C.H. Frotscher (ed.), Marc-Antoine Muret, Opera omnia, vol. 3 (Lipsiae, 18341841), 138 (Var. 7.3.138). Pour chaque référence des Variae lectiones [désormais Var.], sont donnés successivement le numéro du livre, celui du chapitre et éventuellement celui de la page correspondante du troisième volume de cette édition, où se trouvent les Var. Dans le cas des citations, le texte de l'édition Frotscher a été reproduit, tant pour la graphie que pour la ponctuation; afin de faciliter la compréhension du texte latin, j'ai ajouté des guillemets pour signaler au lecteur les passages au style direct.

2 Les travaux sur Muret se sont considérablement accrus ces dernières années. Pour s'en faire une idée, on pourra consulter la bibliographie établie par V. Leroux (ed.), M.-A. Muret, Iuvenilia (Genève, 2009), 502-509. Voir aussi les deux monographies consacrées à l'humaniste: C. Dejob, Marc-Antoine Muret. Un professeur français en Italie dans la seconde moitié du xvie siècle (Paris, 1881) et J.-E. Girot, Marc-Antoine Muret. Des Isles fortunées au rivage romain (Genève, 2012). Il faut enfin signaler la parution récente d'un collectif consacré à Muret: L. Bernard-Pradelle, C. de Buzon et al. (ed.), Marc Antoine Muret, un humaniste français en Italie (Genève, 2020). Les miscellanées de Muret ont peu retenu l'attention de la critique toutefois. L'étude la plus complète sur ce recueil demeure à ce jour le mémoire de master de M. Roux, Les Variae lectiones de Marc-Antoine Muret: l'esprit d'un homme, l'esprit d'un siècle, 2 vol. (Villeurbanne, 2011). On pourra se reporter en outre à J.-E. Girot, "Muret ou l'otium du philologue", in P. Galand-Hallyn, F. Hallyn et al. (ed.), La philologie humaniste et ses représentations dans la théorie et dans la fiction, vol. 2 (Genève, 2005), 527-544; T. Vigliano, "Autres plaisirs de l'écriture philologique dans les Variae lectiones de Marc-Antoine Muret”, in Bernard-Pradelle, Buzon et al. 2020 (comme dans la n. 2), 477-495. 
composition du recueil qu'il lui dédie, Muret insiste sur le caractère inné de son travail de lecture critique, avec des expressions telles que "je suis une inclination de ma nature" ("naturae meae propensionem sequor"), “j' obéis à ma nature” ("naturae meae pareo") ou encore "je pratique cette activité; si on me l'interdisait, je penserais que ma vie n'est pas une vie" ("idque facio, quod si facere vetarer, vitalem mihi esse vitam non putarem") (Var. praef. 4). La philologie paraît ainsi constituer un impératif vital, consubstantiel à l'existence de l'humaniste. Après avoir posé au seuil de ses Variae lectiones cette exigence, Muret ne surprend pas en accordant une place importante à l'écriture à la première personne dans son ouvrage: conséquence du lien établi entre existence et philologie, le "je" y apparaît à chaque page, au risque de la saturer quelquefois. JeanMarc Chatelain a d'ailleurs pu relever la "charge subjective" intrinsèque à ce genre savant. ${ }^{3}$ Le geste du philologue devient ainsi un geste d'écriture de soi, en un siècle qui voit naître la première véritable autobiographie, le De propria vita de Jérôme Cardan, ${ }^{4}$ les Essais de Montaigne ou encore les Ephemerides d'Isaac Casaubon. ${ }^{5}$ En outre, Pétrarque ou Érasme ont déjà ouvert la voie et montré que, par le commerce avec les Anciens, qu'il émende et commente, l'humaniste peut se découvrir et se dévoiler. ${ }^{6}$ Le présent article cherchera donc à cerner la nature du "je" murétien, omniprésent dans les Variae lectiones, à partir de l'analyse d'extraits

3 J.-M. Chatelain, "Les recueils d'adversaria aux XVIe et XVIIe siècles: des pratiques de la lecture savante au style de l'érudition", in F. Barbier, A. Parent-Charon et al. (ed.), Le Livre et l'historien. Mélanges H.-J. Martin (Genève, 1997), 169-186, à 184.

4 E. Wolff (ed.), J. Dayre (tr.), Jérôme Cardan, Ma vie (Paris, 1936), 6. Voir aussi J.Y. Boriaud (ed., tr.), Jérôme Cardan, Le livre de ma vie / De vita propria (Paris, 2020).

5 J. Russell (ed.), Isaac Casaubon, Ephemerides (Oxford, 1850). Les Ephemerides ont été étudiées par H. Parenty, Isaac Casaubon helléniste (Genève, 2009), 207-244.

6 M. Guglielminetti, Memoria e scrittura. L'autobiografia da Dante a Cellini (Torino, 1977), 123-139; L. Hermand-Schebat, Pétrarque épistolier et Cicéron. Étude d'une filiation (Paris, 2011), 411-426; A. Vanautgaerden, "Croire à tout, croire à rien. La question du style dans les lettres-préfaces d'Erasme à son édition de saint Jérôme (Bâle, Jean Froben, 1516)", in D. de Courcelles (ed.), Philologie et subjectivité (Paris, 2002), 5377. Voir également, en guise d'introduction aux questions soulevées par l'écriture à la première personne à la Renaissance, les études suivantes: A. Buck (ed.), Biographie und Autobiographie in der Renaissance (Wiesbaden, 1983); D. de Courcelles 2002 (comme dans la n. 6); S. Gambino Longo, "Autobiographie et auctorialité dans l'écriture encyclopédique de Boccace. L'exemple du De montibus", in H. Casanova-Robin, S. Gambino Longo et al. (ed.), Boccace humaniste latin (Paris, 2017), 109-128; R. Gorris Camos, A. Vanautgaerden (ed.), L'auteur à la Renaissance. L'altro che è in noi (Turnhout, 2009); J. IJsewijn, "Humanistic Autobiography", in E. Hora, E. Kessler (ed.), Studia humanitatis. Ernesto Grassi zum 70. Geburtstag (München, 1973), 209-219; M. Jeanneret, "Je lis, donc je suis: herméneutique et conscience de soi à la Renaissance", in O. Pot (ed.), Émergence du sujet. De l'Amant vert au Misanthrope (Genève, 2005), 151-169; C. Taylor, Sources of the Self: the Making of the Modern Identity (Cambridge, 1989). 
représentatifs de cette somme imposante de dix-neuf livres. L'écriture à la première personne semble y revêtir trois formes. Il existe tout d'abord un "je" qui correspond, pour le dire simplement, à l'implication de l'auteur Muret dans son œuvre. Mais le "je" des Variae lectiones peut renvoyer aussi, bien souvent, à une persona érudite et lettrée qui résulte d'une construction qu'il conviendra d'interroger et dont le but est de consolider le rayonnement de Muret à une période cruciale de sa carrière ${ }^{7}$ - au moment de la première édition des Variae lectiones, en $1559,{ }^{8}$ Muret est installé depuis cinq années seulement en Italie: le Français a encore bien des choses à prouver au-delà des Alpes. Enfin, tout en s'écrivant en philologue, Muret laisse parfois entrevoir un "je" plus personnel, plus intime, qui affleure dans quelques récits d'anecdotes ou d'épisodes vécus par l'humaniste. Ce sont ces trois strates du "je" murétien qui méritent d'être examinées, ainsi que la manière dont elles nourrissent mutuellement l'écriture des Variae lectiones.

\section{L'expression d'une auctoritas}

Le "je" de l'auteur Muret est peut-être le plus facile à identifier. Il est d'abord le "je" du critique, qui formule son avis et se prononce sur la lettre et l'interprétation des textes anciens, d'une manière conventionnelle dans les ouvrages savants. Muret ne déroge pas à cette pratique: l'emploi de la première personne du singulier est fréquemment associé à des verbes de jugement dans les Variae lectiones. Les exemples pourraient être multipliés; je me contenterai de celui du chapitre 3.8 , dans lequel Muret s'interroge sur la gens de Verrès. Le chapitre s'ouvre sur la thèse qui sera discutée par Muret, en l'occurrence la question de savoir si Verrès est issu de la gens Cornelia, thèse discutée contre des individus présentés comme des spécialistes de l'Antiquité romaine ("a quibusdam Romanarum antiquitatum peritissimis hominibus traditum est") (Var. 3.8.59). À l'opinion de ces érudits compétents, mais anonymes, Muret oppose son jugement singulier: "il n'est pas possible de me convaincre" ("mihi persuaderi non potest") (Var. 3.8.60). Il prend en charge toute l'argumentation à la première personne, en recourant à des expressions

7 Sur cette notion, voir J. Lecointe, L’idéal et la différence. La perception de la personnalité littéraire à la Renaissance (Genève, 1993).

8 M.-A. Muret, Variarum lectionum libri VIII (Venetiis, 1559 [USTC 843758]). Une édition augmentée de sept livres (livres 9-15) est imprimée à Anvers en 1580 [USTC 440812]. Les livres 16-19, posthumes, sont publiés à Augsbourg en 1600 [USTC 674279]. 
telles que "mihi videntur", "ego videor affirmare posse", "fieri potest ut fallar" (deux occurrences), "existimo", "arbitror" et "opinor", avant de conclure que Verrès ne peut pas appartenir à la gens Cornelia (Var. 3.8.60-61) - à raison, puisqu'on sait que Verrès est liée à la gens Licinia. Le "je" se charge ici de trancher entre plusieurs hypothèses.

Un emploi proche peut s'observer dans les déclarations de nature méthodologique que Muret distille au gré des Variae lectiones. Au moment de soumettre ses corrections textuelles, l'humaniste les agrémente parfois de quelques remarques théoriques sur la façon dont il conçoit sa tâche de correcteur, comme au début de ce chapitre dédié à l'émendation d'un passage du De bello Gallico de Jules César:

Voici longtemps que j'ai remarqué que de nombreux endroits, chez quelques excellents auteurs, étaient si clairement corrompus que, à mes yeux, c'était la raison même pour ne pas faire de déclaration ouverte sur la façon dont ils semblaient devoir être corrigés, car je me disais que personne ne serait à ce point demeuré pour ne pas voir cela par lui-même: et je formulerais un mauvais jugement sur les talents d'autrui, me semblait-il, si j'estimais que, sans mes avis, on ne remarquerait même pas ces difficultés qui sautaient aux yeux. En réalité, puisque je vois que la plupart des passages de ce genre restent accrochés et fixés même dans ces livres qui paraissent chaque jour avec les émendations des hommes les plus savants, je ne pense pas devoir dissimuler la chose plus avant, étant donné surtout que l'accord de ce si grand nombre d'hommes dotés d'un savoir beaucoup plus grand et d'un esprit critique beaucoup plus vif que les miens me pousse parfois à me demander avec hésitation si je me flatte trop moi-même ou si je suis le seul à avoir la vue brouillée devant les passages mêmes à propos desquels je me dis que les autres manquent de perspicacité.

Iam pridem animadverti multa in optimis quibusque scriptoribus ita manifesto depravata, ut ea ipsa mihi caussa fuerit, ne publice testarer, quomodo emendanda viderentur, quod neminem ita hebetem arbitrabar fore, quin id per se videret: maleque mihi videbar iudicaturus de ceterorum ingeniis, si ne illa quidem, quae in oculos incurrerent, nisi monente me, animadversum iri existimarem. Nunc, cum huius generis pleraque illis quoque in libris haerere ac residere videam, qui quotidie emendati ab hominibus doctissimis prodeunt, dissimulandum amplius non puto: cum praesertim in illa tanta 
hominum multo maiori, quam ego sum, doctrinae copia multoque acriori iudicio praeditorum consensio interdum addubitare me cogat, ne mihi nimis ipsemet blandiar, neve solus is ipsis in locis caecutiam, in quibus alios parum esse arbitror perspicaces. (Var. 10.15.230-231)

L'extrait déborde de marques de la première personne, qui s'oppose, ici encore, à la masse indistincte des autres savants. L'entreprise philologique des Variae lectiones permet à Muret de s'ériger en garant d'un savoir, garant qui n'est pas moins légitime que ses contemporains, et de revendiquer sa propre auctoritas. Au moyen des termes "ipsemet" et "solus", et avec quelque coquetterie, Muret se définit en réaction aux autres ("alios").

Ce "je" auctorial n'apparaît pas néanmoins inconsistant ou sans épaisseur: il s'incarne dans un être de chair et d'os, grâce à des références précises à certains des travaux érudits de Muret, qui n'oublie pas de rappeler au fil des Variae lectiones qu'il est un éditeur de textes réputé et un professeur recherché. Ce sont tout d'abord ses éditions imprimées de textes anciens que l'humaniste évoque ici et là. Les Variae lectiones se font ainsi le reflet de sa production éditoriale, tout en la prolongeant. ${ }^{9}$ Par exemple, en 1.7, Muret revient sur l'épithète "bimaris" qu'Horace applique à Corinthe dans ses Odes (Hor. Carm. 1.7.2), en précisant avoir négligé de la signaler dans son édition commentée du poète latin, parue en 1555 à Venise chez Paul Manuce et dédiée à Jean d'Avanson: ${ }^{10}$

Il a semblé bon d'ajouter une note à ce sujet, puisque dans mes scholies à Horace, que $\mathrm{j}$ 'avais adressées il y a quatre ans au très illustre Jean d'Avanson, qui était alors ambassadeur du roi de France à Rome, comme j'avais signalé l'origine des épithètes de toutes les autres villes, il est arrivé, je ne sais comment, que celle-ci a été omise - ce qui ne convenait pas du tout.

Hoc eo annotare visum est, quoniam in scholiis Horatianis, quae abhinc annos quatuor ad Ioannem Avansonium virum clarissimum, qui tum Romae Gallorum regis legatus erat, miseram, cum

9 L'information de ce paragraphe doit beaucoup à Roux 2011 (comme dans la n. 2), vol. 1,138 .

10 Marc-Antoine Muret, Alde Manuce, Horatius. M. Antonii Mureti in eundem annotationes. Aldi Manutii de metris Horatianis. Eiusdem annotationes in Horatium (Venetiis, 1555 [USTC 835904]). 
ceterarum urbium epitheta unde sumpta essent, indicassem, nescio quo modo contigit, ut hoc, quod minime decuerat, praetermitteretur. (Var. 1.7.14)

Le chapitre des Variae lectiones vient poursuivre la réflexion menée dans une édition antérieure. Un procédé identique s'observe en 5.2, où Muret ouvre sa leçon par la mention de son édition des scholies à Tibulle parue l'année précédente, ainsi qu'en 7.11, où est citée son édition des scholies à Properce, contemporaine de cette dernière. ${ }^{11} \mathrm{Il}$ s'agit probablement pour Muret d'intéresser le public italien à ses travaux récents sur les élégiaques latins, quand, dans les livres plus tardifs des Variae lectiones, est souligné le caractère visionnaire de ses productions de jeunesse parisiennes. Ainsi, en 10.18, Muret évoque sa toute première publication, ses annotations à l'Andrienne et à l'Eunuque de Térence, publiées par Michel de Vascosan en $1551 .{ }^{12}$ À propos du sens de venustas et de invenustus chez Térence, qu'Adrien Turnèbe a plus tard discuté dans ses Adversaria, Muret fait une remarque cinglante:

Ainsi [Turnèbe] a établi que venustas se dit du bonheur qui existe chez les amants et invenustus de celui qui est trop peu heureux en amour, ${ }^{13}$ comme s'il avait été le premier à faire ces remarques, alors que, moi, j'avais enseigné ces mêmes choses environ trente ans plus tôt dans quelques-unes de mes scholies à Térence, que Michel de Vascosan publia à cette époque-là à Paris.

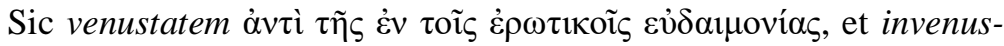
tum pro eo, qui parum in amore felix esset, tanquam a se primum animadversa prodidit, cum ego ante annos plus minus triginta eadem docuissem in Scholiis quibusdam meis in Terentium, quae tum edidit Lutetiae Michael Vascosanus. (Var. 10.18.235) ${ }^{14}$

Muret célèbre la lucidité de ses travaux passés, en invitant peut-être son lecteur à y revenir. L'œuvre de jeunesse n'équivaut pas à un ouvrage de

11 Marc-Antoine Muret, Catullus et in eum commentarius M. Antonii Mureti. Ab eodem correcti et scholiis illustrati Tibullus et Propertius (Venetiis, 1558 [USTC 821189]).

12 Marc-Antoine Muret, In Andriam Terentii annotationes (Parisiis, 1551 [USTC 196402]) et In Eunuchum Terentii annotationes (Parisiis, 1551 [USTC 196405]).

13 Adrien Turnèbe, Adversariorum tomus secundus duodecim libros continens, 22.8, f. 220v (Parisiis, 1565 [USTC 198806]).

14 Voir aussi Var. 18.6. 
débutant: les talents du philologue s'y manifestent déjà. L'exemple précédent souligne en outre à quel point le "je" se plaît à polémiquer contre ses pairs: Turnèbe ne bénéficie nullement d'un traitement de faveur. Muret formule avec régularité des critiques dirigées contre Piero Vettori, l'auteur le plus cité dans les Variae lectiones,${ }^{15}$ ou des blâmes plus ponctuels adressés à d'autres humanistes, contemporains ou non (car la dispute peut aussi s'opérer de manière diachronique chez lui): ${ }^{16}$ Servius (Var. 4.16 et 18.17), Marsile Ficin (Var. 5.4 et 8.4), Érasme (Var. 9.11.202), Guillaume Budé (Var. 4.19.92), Pietro Crinito (Var. 13.8), André Alciat (Var. 9.11.202 et 18.9), Adrien Turnèbe (Var. 4.3, 10.18, 11.19, 17.17 et 17.18), Denys Lambin (Var. 2.17 et 18.9) ou Juste Lipse (Var. 11.1) se trouvent écorchés par Muret, qui crée une rivalité lui permettant d'affirmer son auctoritas. ${ }^{17}$

L'auteur Muret évoque également son activité de professeur à la première personne. Au chapitre 13 du livre 8 , il mentionne ses souvenirs pédagogiques du cours qu'il a donné à Paris en 1551 sur l'Éthique à Nicomaque:

Je me souviens que, il y a huit ans, alors que j'expliquais publiquement ce livre à Paris, je disais que la leçon de ce passage me semblait erronée, de telle sorte cependant que l'on pouvait la corriger sans fournir un gros travail.

15 Notamment en Var. 1.2, 1.13, 1.16 (la critique est accompagnée d'un éloge de Vettori), 2.8, 2.9, 4.5, 4.19, 8.6, 17.4 et 17.13. Voir Roux 2011 (comme dans la n. 2), vol. 1, 193-205.

16 Voir par exemple L. Claire, "Ut notat Politianus in Miscellaneis: Marc-Antoine Muret et Politien", Camenae 22 (2018), 1-15, à 9-13.

17 L'existence de Muret déborde de polémiques avec ses contemporains autour de questions philologiques; quelques-unes d'entre elles trouvent un terrain d'expression dans les Variae lectiones. Sur Muret et Lambin, à propos d'Horace en particulier, se reporter à N. Pace, "Muret e Orazio", Annali online di Ferrara Lettere 11.1 (2016), 4-24 (doi: 10.15160/1826-803X/1284) et à V. Leroux, "Le modèle des Odes d'Horace dans les œuvres poétiques et philologiques de Marc-Antoine Muret", in M. Laureys, N. Dauvois et al. (ed.), Non omnis moriar. Die Horaz-Rezeption in der neulateinischen Literatur vom 15. bis zum 17. Jahrhundert. La réception d'Horace dans la littérature néo-latine du XVe au XVIIe siècle. La ricezione di Orazio nella letteratura in latino dal XV al XVII secolo (Deutschland - France - Italia), vol. 2 (Hildesheim - Zürich - New York, 2020), 933-955, à 942-943; voir aussi Roux 2011 (comme dans la n. 2), vol. 1, 205-220. Sur le différend entre Muret et Lipse au sujet de Tacite, voir L. Claire, "Modalités et enjeux de la polémique autour de Tacite dans la correspondance de Juste Lipse et de Marc-Antoine Muret", in É. Gavoille, F. Guillaumont (ed.), Conflits et polémiques dans l'épistolaire (Tours, 2015), 485-502. 
Memini me, octo abhinc annos, cum eum librum Lutetiae publice interpretarer, ita dicere, mendosam mihi eius loci scripturam videri; sic tamen, ut non magno negotio corrigi posset. (Var. 8.13.177-178)

Muret évoque ensuite sa lecture de l'Andrienne et de l'Eunuque, réalisée la même année (Var. 10.18.235). Plus loin, en 12.15, il fait référence à un cours donné sur le Pro Archia, alors qu'il enseigne au Studium Urbis. Enfin, en 13.12, le cours prononcé sur le De providentia de Sénèque, que l'humaniste a expliqué à Rome en 1574-1575, donne l'occasion à Muret de se représenter en actes, en relatant en détail une saynète fort savoureuse avec l'un de ses auditeurs:

J'expliquais publiquement il y a peu de temps le livre de Sénèque, dans lequel ce vénérable philosophe se demande pourquoi les malheurs s'abattent sur les gens de bien, puisqu'il existe une Providence. Alors qu'on en était venu à la section où l'écrivain lui-même poursuit sans relâche le luxe de son époque, je fus amené, au moment de traiter de ce passage, à énumérer aussi les nombreuses espèces d'oiseaux exotiques que les Romains de l'Antiquité avaient l'habitude de faire venir des régions les plus reculées de toute la terre pour les placer sur leurs tables. Trois jours plus tard, un de ceux qui avaient alors assisté au cours vint me trouver, un homme à ses propres yeux très érudit, et aux miens non dépourvu d'érudition. Ce dernier, comme il avait vu certains de mes proches en user avec moi parfois, avec discrétion certes, mais de façon cependant à en révéler l'artifice, s'appliqua d'abord à faire peu à peu dévier notre discussion, entreprise à propos d'autres sujets, vers l'évocation du discours que j'avais tenu trois jours plus tôt. Alors, comme s'il avait trouvé l'occasion de faire parade de son érudition, il dit: "Je ne voudrais pas que tu aies omis l'autre jour d'évoquer un oiseau, grâce auquel on pouvait concevoir, et même au plus haut point, l'appétit insatiable des anciens dans leur quête de mets raffinés." "Lequel, s’il te plaît?" dis-je. "Le phénix", répondit celui-ci. "Je t'en prie, ces hommes mangeaient-ils aussi du phénix?" "Assurément", dit-il.

Interpretabar nuper publice librum Senecae, quo sanctus ille philosophus quaerit, quare mala bonis viris accidant, cum sit providentia. Cumque ventum esset ad eam partem, in qua ipse graviter insectatur luxum temporum suorum, accidit, ut ego quoque in eo loco 
tractando enumerarem multa genera peregrinarum avium, quas veteres Romani e remotissimis totius orbis partibus petitas mensis inferre soliti essent. Venit ad me triduo post unus eorum, qui tum interfuerant, homo et suo iudicio eruditissimus, et meo non ineruditus. Isque cum aliquot e familiaribus meis mecum una esse vidisset, dissimulanter quidem, sed ita tamen, ut artificium appareret, coepit operam dare, ut sermo noster aliis de rebus susceptus in mentionem eius disputationis, quae triduo ante a me habita erat, sensim delaberetur. Tum quasi occasionem nactus ostentandae eruditioni suae, "Nollem", inquit, "a te tum praetermissam unius avis mentionem, ex qua una inexplebilis veterum libido in exquisitis dapibus conquirendis vel maxime cognosci poterat." "Quam, quaeso?" inquam. "Phoenicem", inquit ille. "Obsecro te, etiam phoenicem illi esitabant?" "Certe”, inquit. (Var. 13.12.295-296)

Le dialogue se prolonge encore sur quelques lignes, à propos desquelles il suffit de savoir que Muret sort vainqueur de la joute verbale face au maître cuistre, qui croit pouvoir en remontrer à un professeur au faîte de sa carrière. À cet interlocuteur sûr de son érudition, pourtant superficielle, étayant son argumentation sur un passage de Plutarque qui aurait recommandé de manger de la cervelle de phénix, selon la traduction latine d'Érasme et de Xylander, Muret oppose sa sagacité philologique et sa connaissance profonde de la langue grecque: les deux traducteurs ont ignoré qu'en grec poĩvi $\xi$ pouvait aussi désigner une espèce de palmier, et

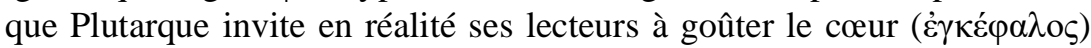
de cet arbre et non la cervelle d'une créature fabuleuse (Plut. Mor. 133.C). Si le récit de cette plaisante anecdote permet d'observer l'esthétique de la varietas et du désordre caractéristique du genre des miscellanées, ${ }^{18}$ il fournit également à Muret l'occasion de faire montre de ses compétences. Le "je", par le rappel régulier de ses titres d'éditeur de textes anciens et de professeur, par le renvoi à d'autres ouvrages de sa composition, assied son auctoritas. Il résulte, par ailleurs, d'une construc-

18 Sur cette esthétique, voir Chatelain 1997 (comme dans la n. 3); P. Laurens, "La poétique du Philologue: les Miscellanea de Politien dans la lumière du premier centenaire", Euphrosyne 23 (1995), 349-367; J.-M. Mandosio, "La miscellanée: histoire d'un genre", in D. de Courcelles (ed.), Ouvrages miscellanées et théories de la connaissance à la Renaissance (Paris, 2003), 7-36; R. Mouren, "La varietas des philologues au XVIe siècle: entre varia lectio et variae lectiones", in D. de Courcelles (ed.), La varietas à la Renaissance (Paris, 2001), 5-31. 
tion éminemment maîtrisée: au fil des Variae lectiones, Muret se forge une persona séduisante.

\section{S'écrire en philologue}

Que ce soit dans la solitude de l'activité intellectuelle ou le tourbillon romain, Muret n'a de cesse de s'écrire en philologue. Si l'auteur est donné, son double littéraire est réfléchi et fabriqué avec art: il s'agit bien de créer "une personnalité apparente susceptible de lui attirer les faveurs [des lecteurs]", pour reprendre les mots de Jean Lecointe. ${ }^{19}$ Quelques brefs passages présentent l'humaniste en train d'étudier dans sa bibliothèque, lisant des textes latins et grecs à l'écart du bruit du monde. Belle illustration du principe vital qu'il a établi dans la préface dédiée à Hippolyte d'Este:

Moi, pendant les heures de l'après-midi, le temps que la chaleur s'atténue, je pénètre dans ma bibliothèque, je m'empare du premier livre qui me tombe sous la main et, en observant si j'y ai apposé un jour quelque remarque, je mets en forme l'un ou l'autre de ces chapitres.

Ego pomeridianis horis, dum se calor frangat, ${ }^{20}$ in bibliothecam meam ingressus, qui primus liber in manus venit, eum arripio, et, si quid olim a me in eo notatum sit inspiciens, unum aut alterum horum capitum concinno. (Var. 16.10.370)

Muret entrouvre ainsi la porte de sa bibliothèque et de son univers intellectuel. Il fait apercevoir à son lecteur son monde privé, où le travail du philologue se fait parfois besogneux et acharné: plus loin, Muret parle de l'épuisement que lui procure la lecture de Plaute (Var. 17.18.416). Ailleurs, il évoque la difficulté qu'il a à retrouver un passage du huitième livre des Deipnosophistes d'Athénée et comment il a été contraint de relire le livre tout entier:

Aujourd'hui donc, comme je devais chercher un passage dans le huitième livre de cet écrivain précisément et que je ne pouvais le

19 Lecointe 1993 (comme dans la n. 7), 385.

20 Souvenir de Cic. De or. 1.265. 
trouver aussi facilement que je l'avais pensé, j'ai pensé qu'il serait préférable de parcourir l'intégralité du livre et de recueillir en passant les remarques dont je jugerais qu'elles pourraient plaire aux savants qui se consacrent à cet auteur, si j'en trouvais.

Hodie igitur, cum mihi ex octavo ipsius libro quiddam petendum esset, neque id tam facile, quam putaram, reperire possem, satius putavi totum librum percurrere, et in transcursu colligere, si qua essent, quae illius studiosis iucunda fore arbitrarer. (Var. 19.3.451)

L'adverbe temporel hodie fait glisser le chapitre vers une forme de chronique ou de journal intime des lectures de Muret, qui offre à son lecteur un bref récit de ses mésaventures quotidiennes, sans doute partagées un jour ou l'autre. Qui en effet ne reconnaît pas ici une situation qu'il a lui-même dû subir? La mention de cette expérience, qu'on imagine agacée, crée ainsi une complicité, éphémère, entre Muret et son lecteur.

Mais le labeur du philologue n'est pas toujours solitaire: le "je" raconte aussi ses amitiés et ses relations. Les Variae lectiones accordent ainsi une belle place à Paul Manuce. Dès le chapitre 6 du premier livre, à propos d'un passage du Pro Quinctio de Cicéron, que Manuce a brillamment édité aux dires de Muret, l'humaniste s'engage dans un éloge plus que chaleureux: "Au sujet de cet homme, assurément, on peut se demander si c'est lui-même qui doit plus à Cicéron ou Cicéron à luimême" ("de quo viro merito dubitari potest, plusne ipse Ciceroni, an ipsi Cicero debeat”) (Var. 1.6.12). La célébration de l'ami se poursuit sur quelques lignes; Muret interrompt sa digression pour l'expliquer par la force du sentiment qui le lie à l'éditeur et imprimeur vénitien:

Mais le feu de l'amour, pour ainsi dire, m'a détourné de mon propos initial. Or, à la vérité, quand il faut parler de Paul Manuce, je ne veux, ni ne peux, ni ne dois réfréner mes paroles.

Sed me amoris quidam aestus ab eo, de quo dicere coeperam, abduxit. Omnino autem, cum de Paulo Manutio loquendum est, moderari orationi meae neque volo, neque possum, neque debeo. (Var. 1.6.13)

Muret donne à voir toute la violence d'un sentiment passionné, si puissant qu'il en devient incontrôlable. Le nom de Paul Manuce surgit encore à plusieurs endroits des Variae lectiones: en 5.11, Muret rapporte un 
jugement émis par Manuce lors d'une discussion et prolonge dans la leçon la conversation tenue avec son ami; en 10.20, il convoque ses souvenirs personnels à propos de la correction d'un passage du De bello Gallico, tout en procédant à son propre éloge de manière oblique:

Je me souviens que j'avais dit cela même à Paul Manuce, quelques années auparavant, et que ce dernier avait approuvé avec conviction ma conjecture.

Memini me hoc ipsum ante aliquot annos Paulo Manutio dicere eique vehementer probatam esse coniecturam meam. (Var. 10.20.237)

Un autre nom revient souvent dans les Variae lectiones: celui du cardinal Hippolyte d'Este, le protecteur de Muret. Si la relation de dépendance qui existe entre les deux hommes peut faire peser quelque soupçon de complaisance sur les louanges que Muret adresse à Hippolyte, Muret réussit à recréer l'ambiance d'amitié et d'estime réciproques qui avait cours à Tivoli, en faisant revivre ses tête-à-tête familiers avec Hippolyte:

Quand nous nous étions retirés à Tivoli, où celui-ci avait l'habitude de passer chaque année les mois d'été, aucun jour ou presque ne s'écoulait sans que, sous couleur d'étudier, après avoir renvoyé tous nos autres compagnons, nous occupions quelques heures à discuter ensemble de la manière la plus agréable.

Cum Tibur secesseramus, ubi ille quotannis aestivos menses transigere solebat, nullus fere abibat dies, quin per studiorum speciem, aliis omnibus exclusis, horas aliquot una iucundissimis sermonibus consumeremus. (Var. 16.4.365)

Dans le même chapitre, Muret rappelle aussi un déjeuner en compagnie d'Hippolyte et d'autres convives dans la villa de son protecteur, au cours duquel a été débattue avec vivacité la question de la rigueur morale des princes. Muret s'y dépeint au milieu d'une société joyeuse et cultivée: bien que l'étude soit indispensable, le philologue ne saurait faire vœu d'érémitisme. Il faut noter que Muret aime à raconter des anecdotes personnelles dans lesquelles il se trouve entouré d'un public, comme en 10.7: 
Un jour, alors que j'étais tranquillement installé dans quelque boutique de livres et que j'y discutais avec plusieurs amis, des hommes savants, me tombèrent sous la main les remarques faites sur Cicéron par quelque individu, assurément cultivé et consciencieux, ${ }^{21}$ dans lesquelles, à un certain endroit, il condamne avec virulence le jugement de nous tous sur cette tournure et soutient qu'il faut ajouter une négation à tous ces passages que nous utilisions pour la confirmer. ${ }^{22}$

Inciderunt nuper in manus meas, cum in taberna quadam libraria otiosus sederem ibique cum amicis aliquot eruditis hominibus colloquerer, notae cuiusdam sane docti ac diligentis viri in scripta Ciceronis, in quibus quodam loco vehementer nostrum omnium in eo iudicium improbat, et illis omnibus locis, quibus nos ad id confirmandum utebamur, negationem addendam esse contendit. (Var. 10.7.220-221)

Ces scènes de sociabilité, où le moi évolue en nous, font partie de l'identité des Variae lectiones, une sociabilité dans laquelle la place de Muret est toujours centrale: tantôt celle d'un primus inter pares, comme à Tivoli ou dans l'échoppe du libraire, tantôt celle du maître qui domine, comme lorsqu'il reçoit à son domicile la visite d'une délégation d'étudiants venus d'Allemagne. Le premier chapitre du quinzième livre des Variae lectiones s'ouvre ainsi:

Dans le but de me rencontrer et de me saluer, quelques jeunes gens de bonne famille, originaires d'Allemagne, étaient venus me voir, dans ma maison du Quirinal. Alors qu'ils avaient parcouru quasiment toute l'Italie et qu'ils n'avaient pas manqué de voir chacun ou presque des hommes qui possèdent quelque renom dans les lettres, ils avaient voulu me voir aussi pour la même raison, comme euxmêmes le disaient en tout cas, et discuter avec moi.

21 Henri II Estienne, Pseudocicero dialogus ([Genevae], 1577 [USTC 450742]), 216.

22 L'usage cicéronien en question est celui de l'expression "non modo" pour "non modo non", que Paul Manuce et, à sa suite, Muret ont remarquée chez l'Arpinate. Voir P. Manuce (ed.), Cicéron, Epistolae familiares diligentius, quam quae hactenus exierunt, emendatae. Pauli Manutii scholia, quibus et loci familiarium epistolarum obscuriores explanantur, et castigationum, quae in iisdem epistolis factae sunt, ratio redditur (Venetiis, 1540 [USTC 822132]), f. AAA iiii r et DDD iii v; D. Ruhnken (ed.), M. Antonii Mureti opera omnia, vol. 2 (Lugduni Batavorum, 1789), 546. 
Venerant ad me in Quirinalem visendi ac salutandi caussa nobiles aliquot Germani adolescentes, qui cum totam prope Italiam perlustrassent neque quemquam fere praetermisissent eorum, qui nomen aliquot habent in litteris, quin eum vidissent, me quoque, ut ipsi quidem dicebant, eadem de caussa videre mecumque colloqui voluerant. (Var. 15.1.326)

L'extrait montre bien la construction qui sous-tend l'écriture du "je". Même si Muret veille à préciser en incise qu'il se contente de rapporter le discours des étudiants allemands ("ut ipsi quidem dicebant"), l'anecdote qu'il rapporte témoigne d'un art élaboré. Tout d'abord, sa situation au seuil du chapitre et du livre la met doublement en valeur. Ensuite, son récit permet à Muret de rappeler à peu de frais qu'il est l'une des gloires de l'Italie, dont la réputation s'étend même au nord des Alpes. Il se dépeint ainsi en célébrité de l'érudition, une célébrité qui reste néanmoins très accessible, puisqu'il reçoit les étudiants à son domicile personnel: en quelques lignes, l'autoportrait met en avant des qualités d'hospitalité, de bienveillance et de disponibilité dont la grandeur n'a d'équivalent que la renommée et la compétence de Muret.

Si les anecdotes personnelles rapportées par Muret peuvent sembler sortir du cadre des miscellanées et entretenir quelque parenté avec des genres plus autobiographiques, elles soulignent néanmoins toujours les talents de philologue de Muret ou visent à consolider l'image de ce dernier. Dans le cas des moments d'amitié avec Paul Manuce et Hippolyte d'Este, il s'agit sans doute pour Muret de s'installer dans le paysage lettré italien en mettant en valeur son réseau scientifique, voire en l'exhibant. Des considérations de carrière viennent soutenir l'écriture et, en dépit du cadre amical, le propos tenu reste celui d'un homme soucieux de montrer son appartenance à une sodalitas. Dans ce sens, sans doute, fautil comprendre les nombreuses citations de noms de contemporains qui parsèment les Variae lectiones. En particulier, Muret précise souvent le nom de l'ami, de la connaissance ou du cardinal qui lui a prêté, voire offert un manuscrit, comme les cardinaux Paul Farnèse (Var. 9.20.212) et Guglielmo Sirleto (Var. 10.14.230 et 14.8.312), ou les savants Fulvio Orsini (Var. 11.17.258) et Vincenzo Lauro (Var. 12.15.275 et 12.7.268). Pareillement, quand Muret se représente dans la boutique du libraire ou à table à Tivoli, il se dépeint en train de juger des travaux critiques et se montre en figure d'autorité philologique. Même face à son auditeur cuistre, Muret est sollicité pour l'excellence de ses avis et de son jugement. Le "je" vient servir une persona travaillée au plus haut point et 
semble rester sous un contrôle délibéré, en dépit de quelques incursions dans la vie de Muret.

Avec quelque paradoxe, l'hypothèse d'une écriture de soi très maîtrisée se confirme à l'examen de chapitres des Variae lectiones dépourvus de toute marque grammaticale de la première personne, alors que leur thématique rencontre des échos certains avec la vie de l'humaniste. Trois chapitres au moins peuvent dérouter le lecteur averti de la biographie murétienne. Le chapitre 3 du deuxième livre propose une réflexion autour de la sentence "Patria est ubicunque bene est". On pourrait s'attendre à trouver quelques confidences de la part d'un individu qui a tôt quitté sa patrie, qui a connu l'exil et qui n'a pas dédaigné d'aborder ce thème dans sa correspondance, notamment quand, en 1561, il accompagne le cardinal Hippolyte d'Este au colloque de Poissy. À cette occasion, les deux hommes séjournent quelques mois à Paris. ${ }^{23}$ Muret, qui en profite pour fréquenter les cercles intellectuels de la capitale, ${ }^{24}$ demeure aussi à Chaalis, où il se languit de l'Italie. Un extrait de la lettre $29 \mathrm{du}$ premier livre de la correspondance de Muret, datée du 14 novembre 1562 et adressée au médecin d'Hippolyte d'Este, Giacomo Canani, révèle que Muret est contraint de demeurer en France, malgré la hâte qu'il a de rejoindre son ami pour partager de nouvelles lectures:

J'en viens à présent à un autre sujet: nous allons passer l'hiver en France, désormais, le fait est assuré à mon avis; de toute manière, cette situation sera pour moi extrêmement fâcheuse, le temps de pouvoir être à Paris ou dans une autre ville. Car assurément, voilà bien longtemps que je suis fatigué de ces bois-là; ${ }^{25}$ et rien ne me semble plus long que d'attendre d'être à tes côtés, pour que, selon notre habitude, nous fassions ensemble un peu de lecture chaque jour.

Nunc ad alia venio: hybernaturos in Gallia esse nos, iam, ut opinor, exploratum est; aeque id mihi valde molestum erit, dum aut Lutetiae aut in aliquo alio oppido esse liceat. Nam horum quidem nemo-

23 Le cardinal et Muret quittent Rome à l'été 1561, pour y revenir en septembre 1563. Entre ces deux dates, Muret se partage entre Paris et Chaalis. Sur le détail de ce séjour et des activités de l'humaniste, voir Dejob 1881 (comme dans la n. 2), 152-166 et Girot 2012 (comme dans la n. 2), 32-33. $19-22$.

24 Dejob 1881 (comme dans la n. 2), 146-166 et Girot 2012 (comme dans la n. 2),

25 Chaalis. 
rum iam pridem taedet; neque mihi quidquam longius videtur, quam dum tecum sim, ut more nostro legamus una aliquid quotidie. ${ }^{26}$

$\mathrm{Ni}$ déclaration comparable ni plainte élégiaque dans le chapitre des Variae lectiones: Muret préfère évoquer deux figures paradigmatiques de l'exilé dans le monde gréco-romain, Ulysse et Ovide, sans jamais recourir à la première personne ni évoquer son expérience personnelle.

Un phénomène similaire s'observe en 5.1 , chapitre consacré à la description et à l'emplacement du lieu mythique des Îles fortunées. Le chapitre s'étend longuement, en compilant les témoignages d'auteurs anciens, en particulier Homère et le byzantin Jean Tzétzès. Or les "Isles fortunées" sont aussi une fameuse ode que Pierre de Ronsard a dédiée à Muret, qui se trouve dans l'édition de 1553 des Amours nouvellement augmentées et commentées par Muret. ${ }^{27}$ Dans cette ode, Ronsard représente son commentateur entouré d'une vingtaine d'amis (parmi lesquels Jean-Antoine de Baïf, Nicolas Denisot, Joachim Du Bellay, Jean Dorat, Étienne Jodelle ou encore Pierre de Paschal) et le compare à un nouvel Orphée. Le silence de Muret dans son chapitre des Variae lectiones interroge.

Enfin, en 17.8, Muret débat de la question de savoir s'il est utile de voyager. Là encore, la réponse à cette question à laquelle Muret pourrait apporter sa contribution personnelle passe par la mention d'auteurs de l'Antiquité et de la figure d'Ulysse. Toute allusion à son passé et à son réseau français semble volontairement gommée par l'humaniste: le "je" se projette dans sa carrière italienne et la remémoration des souvenirs de jeunesse, en particulier parisienne, apparaît comme un adynaton. Dans ce même chapitre pourtant, l'émotion guette et surgit de manière fulgurante à la lecture de Claudien:

Pour ma part, je suis bouleversé au plus profond de mon être, quand je lis ce petit poème de Claudien [Claud. Carm. 20.21-22], par lequel il décrit ce vieillard de Vérone, qui jamais n'avait quitté la terre de ses ancêtres.

26 C.H. Frotscher 1834-1841 (comme dans la n. 1), vol. 2, 65-66.

27 C. de Buzon, P. Martin (ed.), P. Ronsard, M-A. Muret, Les Amours, leurs Commentaires (Paris, 1999), 264-271. 
Equidem afficior intimis sensibus, cum illud Claudiani poemation lego, quo senex ille Veronensis describitur, qui nunquam e patriis finibus excesserat. (Var. 17.8.402)

À la lecture d'un vers, d'un poème, le "je" s'émeut et l'écrit. Ainsi, la persona de philologue construite par Muret dans les Variae lectiones se fissure de manière éphémère et laisse place à une écriture plus personnelle: pour cela, le stimulus du texte antique a été nécessaire.

\section{Fragments autobiographiques}

De fait, l'émotion pointe parfois au détour d'une leçon. Les quelques passages concernés sont tous liés à la lecture des Anciens: c'est dans l'acte de lecture, antérieur au jugement philologique, que Muret livre un peu de lui-même. Même si ces remarques sont rares à l'échelle de la masse foisonnante des dix-neuf livres des Variae lectiones, l'humaniste peut reconnaître être bouleversé au détour d'une phrase ou d'une correction. Ainsi, en 2.7, Muret parle du bonheur qu'il éprouve à découvrir des parallèles entre la littérature latine et la littérature grecque, comme lorsqu'il décèle que Cicéron imite les orateurs attiques:

Or il me plaît d'observer cela, dans la mesure du possible: et j'avoue que je tire de la confrontation de passages de ce genre une sorte de plaisir singulier en mon for intérieur, et celui-ci, à mon avis, n'est ni vulgaire ni dépourvu de profit.

Iuvat autem me, quatenus licet, id observare: profiteorque, me ex eiusmodi locorum contentione singularem quandam eamque, ut opinor, neque illiberalem, neque frugis expertem capere animo voluptatem. (Var. 2.7.36)

Le verbe "profiteor" et le substantif "animo" placent le "je" sur le terrain de l'intériorité: Muret confesse le sentiment qui naît de la fréquentation des textes anciens. Plus loin, en 14.4, il se décrit riant aux éclats à la lecture du passage de Pline l'Ancien sur le taureau sauvage ("bonasus"), susceptible de catapulter ses excréments à une distance de trois jugères, soient soixante-quinze ares (Plin. M. NH 7.40). Le commerce avec les Anciens est capable de faire tomber le masque du philologue minutieusement élaboré. 
Le rapport à l'activité philologique peut être lui-même décrit en des termes pleins d'affection. Dans le premier chapitre du livre 11 des Variae lectiones, Muret recourt au vocabulaire de l'amour filial et appelle l'une de ses conjectures sa "filiola", adaptant une image topique chez les humanistes, celle du livre-enfant. ${ }^{28}$ Il compare Juste Lipse, le trop zélé éditeur de Tacite, à un époux qui serait venu lui enlever sa fille sans demander l'autorisation paternelle:

Mais rien ne trompa mon attente autant que cela: la conjecture par laquelle moi-même je trouvais grâce surtout à mes propres yeux, que très souvent j'avais montrée à des hommes très érudits, que j'embrassais comme ma petite fille, à savoir qu'on devait lire "Gnarum id Caesari" là où tous avant moi avaient lu sans aucun sens "C. Navum id Caesari” (Tac. Ann. 1.5.2), cette conjecture donc était venue aussi à l'esprit de Lipse. ${ }^{29}$ Or je pensai qu'il m’était arrivé la même chose qu'aux nombreux pères qui, pendant qu'ils gardent trop longtemps à la maison leurs filles adultes et nubiles, et négligent de leur chercher une situation, obtiennent que pendant ce temps, elles se cherchent un mari elles-mêmes.

Sed nihil mihi tam praeter opinionem accidit, quam eam coniecturam, qua ipse mihi praecipue placebam, quam eruditissimis hominibus saepissime ostentaveram, quam ut filiolam amplexabar meam, legendum esse, "Gnarum id Caesari”, ubi ante me omnes nullo sensu legerant, "C. Navum id Caesari”, eam Lipsio quoque in mentem venisse. Cogitavi autem idem mihi evenisse, quod multis patribus, qui, dum adultas ac nubiles filias nimium diu domi habent eisque conditionem quaerere negligunt, efficiunt interdum, ut ipsae sibi maritos quaerant. (Var. 11.1.239-240)

La comparaison filée pour décrire le sentiment éprouvé par Muret lors de la découverte du procédé de Lipse - considéré par Muret comme un vol

28 N. Kenny, "Le livre-enfant, ou les enjeux sociaux et familiaux d'un topos", in I. Salas (ed.), Les métaphores du livre à la Renaissance (à paraître). En attendant la publication de cet ouvrage, issu d'un colloque, on peut consulter la captation vidéo de la conférence de N. Kenny (url: https://live3.univ-lille3.fr/video-etudes/les-metaphores-dulivre-a-la-renaissance-2-2.html).

29 Juste Lipse (ed.), Tacite, Historiarum et Annalium libri qui exstant, Iusti Lipsii studio emendati et illustrati [...]. Eiusdem Taciti liber de moribus Germanorum; Iulii Agricolae vita. Incerti scriptoris dialogus de oratoribus sui temporis (Antverpiae, 1574 [USTC 403285]), 217. 
indélicat - dit quelque chose de la dimension intime du rapport que l'humaniste entretient avec l'activité philologique. ${ }^{30} \mathrm{Ce}$ rapport peut même s'apparenter à une maladie (“morbus"):

J'ai l'habitude de parcourir avec plaisir tous les manuscrits qui me tombent sous la main: en bref, de quelque nature que ceux-ci soient et de quelques caractères d'écriture qu'ils soient composés. Pline avait l'habitude de dire de tous les genres de livres qu'il n'y en avait aucun de si mauvais qu'on ne puisse en tirer quelque profit (Plin. m. Ep. 3.5.10). Pour ma part, je trouve ce mot à leur propos tout à fait vrai. J'en attrape en effet beaucoup qui sont mutilés, déchirés, corrompus, dont les lettres s'effacent; en outre, ce qui est plus gênant, qui sont écrits par des individus extrêmement incompétents, et pour cette raison "regorgeant de blessures", 31 si bien qu'il faut dépenser pour ainsi dire une gêne incroyable quand on les lit. Mais je n'en rencontre pas beaucoup qui peuvent en tout point tromper mon attente. Car cependant, toujours ou presque, je fais un petit gain. Or soit qu'il faille appeler cela de la constance, soit même une sorte d'errance de l'esprit (car je n'empêche personne de l'appeler du nom qu'il voudra), je suis d'un caractère si obstiné qu'aucun nombre d'erreurs n'est assez grand pour me détourner de ma lecture. Et je n'ai pas l'impression d'avoir mal placé mon temps quand, après être sorti de ces difficultés, comme de certains terrains accidentés, je me rends compte au cours de mon examen que l'un ou l'autre passage de quelque bon auteur a retrouvé sa pureté grâce à un travail de deux ou trois jours. Or de nombreux amis, qui savent que je suis atteint de cette maladie, m'envoient de nombreuses pages qu'ils ont recueillies n'importe où.

Soleo libenter veteres libros, quicumque in manus meas incidunt, evolvere: cuicuimodi tandem illi sint et quocunque litterarum charactere exarati. Quod enim Plinius de toto librorum genere dicere solebat, nullum esse tam malum, ex quo non caperetur aliquid fructus: id ego in his maxime verum esse comperio. Multos enim nanciscor mutilos, laceros, corruptos, evanescentibus litteris; tum, quod molestius est, descriptos ab hominibus imperitissimis,

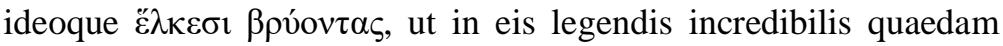

30 Sur le conflit qui opposa les deux hommes, voir Claire 2015 (comme dans la n. 17).

31 R. Kannicht (ed.), Euripide, Fragmenta (Göttingen, 2004), 1086. 
molestia exhaurienda sit. Sed non in multos incido, qui omni ex parte frustrentur opinionem meam. Fere enim semper aliquid tamen lucelli facio. ${ }^{32}$ Sive autem haec constantia vocanda est, sive etiam quidam mentis error ${ }^{33}$ (non enim, quominus quisque quo volet nomine appellet, impedio), ita obstinato animo sum, nulla ut sit tanta erratorum multitudo, quae me a legendo absterreat. Neque mihi male operam collocasse videor, cum ex illis difficultatibus, tanquam e salebris quibusdam, emersus, in recensione reperio, unum aut alterum boni alicuius scriptoris locum bidui triduive labore purgatum esse. Multi autem amici, qui me hoc morbo teneri sciunt, multas ad me schidas undecunque collectas mittunt. (Var. 2.16.45)

La boulimie dont souffre Muret se traduit dans les accumulations qui traduisent un besoin inassouvissable de manuscrits. L'humaniste reconnaît lui-même ce que cette habitude peut avoir de malsain, avec des expressions comme "error animi” ou "obstinato animo". Il détourne, en l'inversant, une image traditionnelle du vocabulaire philologique: ce n'est plus le texte qui est malade ni le correcteur qui soigne, mais ce même correcteur qui est frappé d'un "morbus". ${ }^{34} \mathrm{Du}$ reste, en indiquant être prêt à passer deux ou trois jours à émender un seul passage, Muret révèle assez le rapport de dépendance maniaque qu'il nourrit à l'égard de l'activité philologique.

L'écriture de soi peut aussi toucher à l'intime lorsque Muret évoque son neveu, Marc-Antoine junior, le fils de son frère né en 1570 et dont il a la charge de l'éducation à Rome. Muret prend très à cœur son rôle de tuteur: en 1578, après avoir mené l'enfant en pèlerinage à Notre-Dame de Lorette, il compose à son attention une Institutio puerilis, recueil versifié destiné à lui apprendre le latin. ${ }^{35}$ Les deux chapitres des Variae lectiones consacrés à Marc-Antoine junior témoignent de la profonde tendresse que l'oncle éprouve à l'égard du jeune garçon:

32 Réminiscence de Sen. Ep. 1.5.7.

33 Allusion à Sen. Ep. 1.2.

34 V. Leroux, "Le philologue inspiré", in Galand-Hallyn, Hallyn et al. 2005 (comme dans la n. 2), vol. 2, 343-370, à 355, n. 25, donne quelques exemples de l'usage de cette image chez Muret.

35 Marc-Antoine Muret, Puerile institutione fatta da monsignor Mureto in versi latini et da m. Giulio Durante scolare in Sapienza di Siena ridotta in terza rima (Roma, 1579 [USTC 843754]). Voir Girot 2012 (comme dans la n. 2), 40 et 710. 
L'amour que je ressens pour mes proches me contraint à retomber en enfance. C'est pourquoi, moi qui avais laissé depuis longtemps désormais les comédies et autres amusements, pour me consacrer à des occupations plus sérieuses et plus appropriées à mon âge, à présent que j'ai la charge des études de Marc-Antoine, qui au regard de la nature est le fils de mon frère, au regard de l'affection et des sentiments le mien, je prends de temps à autre entre mes mains Térence, mes amours et mes délices d'autrefois, et je parcours ses pièces avec ce petit garçon qui présente les plus belles espérances, avec un grand plaisir en mon for intérieur.

Repuerascere me cogit amor in meos. Itaque qui iam pridem et comoedias et cetera ludicra posueram, ut graviora et huic aetati convenientiora curarem, nunc ut consulam studiis M. Antonii, qui natura fratris mei filius est, caritate et benevolentia meus, Terentium, amores olim ac delicias meas, sumo interdum in manus, eiusque fabulas cum optimae spei puerulo magna cum animi mei voluptate percurro. (Var. 16.3.362-363)

Multiples sont les sentiments convoqués par l'humaniste, qui mentionne son "amor", sa "caritas", sa "benevolentia" à l'égard du petit garçon ("puerulus"). L'emploi du dimunitif possède une nette valeur hypocoristique. Muret écrit aussi le plaisir ("magna cum voluptate") qu'il prend à initier aux joies de la lecture des Anciens un enfant plus que prometteur et semble céder à une forme de vanité paternelle, qui le pousse à consacrer un peu plus loin dans le recueil un chapitre entier aux exploits philologiques de son neveu:

Je ne priverai pas de sa petite gloire un garçon doué d'un naturel talentueux et plein de finesse, Marc-Antoine, le fils de mon frère, qui aujourd'hui, sur mon ordre, a comparé le premier livre de l'Anabase avec la traduction de Romolo. ${ }^{36}$ Il remarqua d'une part que d'assez nombreux mots avaient été soit omis, soit rendus de manière fort approximative par le traducteur; il remarqua d'autre part ce passage, qui m'a semblé digne d'attention, puisqu'à la fois il

36 On ignore quelle édition de cette traduction utilise Muret; cette dernière fut publiée pour la première fois en 1533: R. Amaseo (tr.), Xénophon, De Cyri minoris expeditione libri VII (Bologna, 1533 [USTC 863994]). 
est connu et n'a pas retenu l'attention d'un excellent imprimeur, ${ }^{37}$ qui par ailleurs pourtant fut assez vigilant en remarquant les erreurs de Romolo.

Non fraudabo gloriola sua scito et eleganti praeditum ingenio puerum, M. Antonium, fratris mei filium, qui hodie iussus a me Xenophontis primum Anabaseos conferre cum interpretatione Romuli, cum alia satis multa notavit ab interprete partim omissa, partim negligentius reddita, tum hoc, quod mihi animadversione dignum visum est, cum et insigne sit, neque ab optimo typographo, qui tamen in notandis Romuli erratis satis alioqui diligens fuit, animadversum. (Var. 17.20.418)

Dans les lignes qui suivent, l'exploit du jeune Marc-Antoine explicitement célébré consiste à avoir remarqué qu'un passage de la traduction de l'Anabase faite par Romolo Amaseo, à la fin de l'éloge de Cyrus, contredit la lettre de Xénophon et se méprend sur les rôles du roi Artaxerxès, d'Orontas et du confident de ce dernier (Xen. An. 1.9.29) - confusion qui a échappé à Henri II Estienne lui-même. La prouesse implicite est aussi celle d'être capable, à douze ans, de lire couramment Xénophon dans le texte grec, d'émettre de pertinents jugements critiques sur la traduction latine réalisée par l'un des grands noms de l'humanisme philologique, Romolo Amaseo, et de surpasser un illustre helléniste, Henri II Estienne, dans la révision de cette dernière. Cette sagacité suscite l'admiration et l'étonnement non dissimulés de l'oncle, avec peut-être quelque fatuité:

Cette remarque, comme elle touchait à la vérité historique, et au plus haut point même, et que, à mon étonnement, elle avait été faite avec une perspicacité extrême par un garçon qui n'avait pas encore douze ans, j’ai jugé digne de la transcrire.

Hoc cum et ad historiae veritatem vel maxime pertineret, et, mirante me, a puero nondum duodecim annos nato sagacissime animadversum esset, dignum, quod adscriberem, iudicavi. (Var. 17.20.418)

37 Henri II Estienne donne une édition bilingue grec-latin de Xénophon en 1561. Pour l'Anabase, il reprend la traduction de Romolo Amaseo, qu'il escorte de ses propres notes en marge: Omnia quae extant opera. In Xenophontem annotationes cum Latina interpretatione ([Genevae], 1561 [USTC 450506]). 
C'est sans doute dans l'expression de cette admiration que Muret donne un accès à son monde intérieur, sans filtre ni recomposition.

L'écriture à la première personne dans les Variae lectiones relève de mécanismes complexes. Si le "je" fait souvent référence à des épisodes de la vie de Muret, la plupart des exemples pris en considération montrent une écriture de soi savamment construite et élaborée: en dépit de quelques passages qui entretiennent une parenté avec le journal intime, l'écriture de soi paraît surtout au service d'une mise en valeur de soi. Dans les Variae lectiones, le "je" qui se déploie avec envergure est celui d'un philologue qui revendique son auctoritas et entend maîtriser son image. En de rares passages néanmoins, le masque se fissure, quand l'émotion suscitée par les textes est trop forte ou quand les talents du petit Marc-Antoine transportent son oncle. Le "je" se dévoile alors à la faveur du commerce avec les Anciens. Mais cette intimité qui sourd ne dure pas et, à la différence d'un Montaigne par exemple, que Muret connaît bien, ${ }^{38}$ et dont les $E s$ sais, contemporains des Variae lectiones, présentent des points de convergence avec la forme des miscellanées, ${ }^{39}$ l'écriture de soi ne vise pas la connaissance de soi. Bien que Muret se raconte, par petites touches, la peinture qu'il donne de lui-même ne constitue jamais le sujet de son propre ouvrage.

Université de Picardie Jules Verne lucie.claire@u-picardie.fr

38 Sur les liens qui unissent Muret à Montaigne, voir R. Trinquet, "Un maître de Montaigne: l'humaniste limousin Marc-Antoine Muret. Sa carrière pédagogique en France”, Bulletin de la société des amis de Montaigne 4.7 (1966), 3-17 et M. Wiesmann, "Muret", in Ph. Desan (ed.) Dictionnaire de Michel de Montaigne (Paris, 2007), 801-802.

39 F. Gray, Montaigne bilingue: le latin des Essais (Paris, 1991), 118. 